\title{
Design of a General Development Framework for Web-based Information
}

\author{
System \\ Zhang Dong ${ }^{1, \mathrm{a}^{*}}$, Lv Yanmei ${ }^{2}$, Niu Gang ${ }^{3}$, Zhen Hongtao ${ }^{4}$ \\ 1,2,3,4 Institute of Machine Technology, Shijiazhuang, China \\ a310792132@qq.com
}

Keywords: Information system,Framework, Middle layer

\begin{abstract}
The paper studies the concept and characteristics of the information system general framework, designs a general development framework of web based information system, and gives the detailed design based on MVC pattern of the middle layer of the framework.
\end{abstract}

\section{The Information System and its Development Framework}

Information system, also called Management Information System, refers to a people-oriented system using for information collection, transmission, processing, storage, updating, expansion and maintenance [1]. The system consists of computer hardware, software, network communication equipment and other office equipment.

In computer technology, the system framework on the application aspect refers to the reusable design of the whole or part of the system, which characterized by a set of abstract components and interaction methods between them.

The use of the framework is quite extensive in the web based system development. Web framework is a software development framework using to support dynamic Web sites, Web applications and Web services development [2]. Web framework based on the bottom language of the server development is to improve the code reusability and to normalize the development process. The framework encapsulates some common operations and functions of the web development.

General framework of information system refers to the specialized software framework used to develop information system framework. At present most of the information system framework are based on web development framework. In order to reduce the workload in the process of the B/S structure system development, the general parts of the system are taken out and in accordance with the requirements of the software engineering considering the implementation needs of information system to form a general system framework.

The information system framework according to the thought of design patterns is designed hierarchically on the basis of the Web development framework. Which eases the burden of the system design and enhances the standardization of development to a certain extent. So the overall cost level of design and development can be reduced and developers focus more on the development of specific business [3].

The information system framework designed in this paper is an B/S structure system framework based on Linux operating system, taking Apache as the HTTP server, PHP as the server-side development language, the Yii Framework as development framework, MySQL as the database management system. The Yii Framework is a high-performance PHP framework based on components, which is used in large web application development. The Yii Framework is in 
accordance with the OOP pattern, which gives perfect library references and comprehensive tutorial. And the framework is one of the most efficient PHP framework offering almost all the required functions of a web 2.0 application development including the MVC, DAO/ActiveRecord, widgets, web services, etc.

\section{Design of the General Framework}

The general framework for information system designed in this paper mainly consists of the middle layer and the general function module. The middle layer based on the Yii framework provides the general modules of the upper layer the function interface. And the general modules encapsulate the common function of information systems as modules. The architecture of the general framework for information system is shown in Figure 1. Each layer of the architecture provides corresponding function interface for upper layer. The paper mainly discusses the design of the middle layer of the framework.

\begin{tabular}{|c|c|c|c|}
\hline \multicolumn{3}{|c|}{ THE APPLICATION LAYER } & BUSINESS LOGIC \\
\hline USER MANAGEMENT & $\begin{array}{c}\text { FUNCTION } \\
\text { ORGANIZATION } \\
\end{array}$ & $\cdots \cdots$ & \multirow{2}{*}{ SYSTEM FRAMEWORK } \\
\hline \multicolumn{3}{|c|}{ THE MIDDLE LAYER } & \\
\hline \multicolumn{3}{|c|}{ Yii Framework } & \multirow{3}{*}{ LANGUAGE FRAMEWORK } \\
\hline \multicolumn{3}{|c|}{ PHP } & \\
\hline Web server & Database & $\ldots \ldots$ & \\
\hline \multicolumn{3}{|c|}{ OS (Linux) } & SYSTEM ENVIRONMENT \\
\hline
\end{tabular}

Figure 1 the general information system framework

\section{Design of the Middle Layer}

The middle layer consists of four main parts including code organization, database access, front-end development and request processing. The front-end web development which has evolved from web page creation, mainly includes front-end graphic, browser compatibility, CSS and HTML interaction design, etc. The request processing completed by the Yii framework. The Yii framework supports automatic generation of complex WSDL service specifications and web service request processing. So this dissertation mainly discusses the middle layer code organization and the database access design.

Code organization. The framework uses the MVC (Model-View-Controller) architecture for system development code organization. The main business logic code of the whole system is divided into three parts according to the specific function.

The model. The model refers to the business process handling and business rules, which implements the mechanism and function of the whole system [4]. The main role of the model is to operate and maintain information and data of the system. In the framework, the model is mainly responsible for interacting with the database. In addition, the model also includes many arithmetic which is closely related with the data object. 
The view. The view is mainly responsible for generating user interface. The framework sends data to the view layer, and the latter call the appropriate template to display the data, or process the data and pack into a response sent to the client for display.

The controller. The controller defines the behavior of the application, and it is responsible for requests interpretation and business processing from users of the view [4]. The controller is responsible for calling the model to obtain and process the corresponding data according to the request and then send to the view to get the final response. In the framework, most codes associated with specific business logic are implemented in the controller layer.

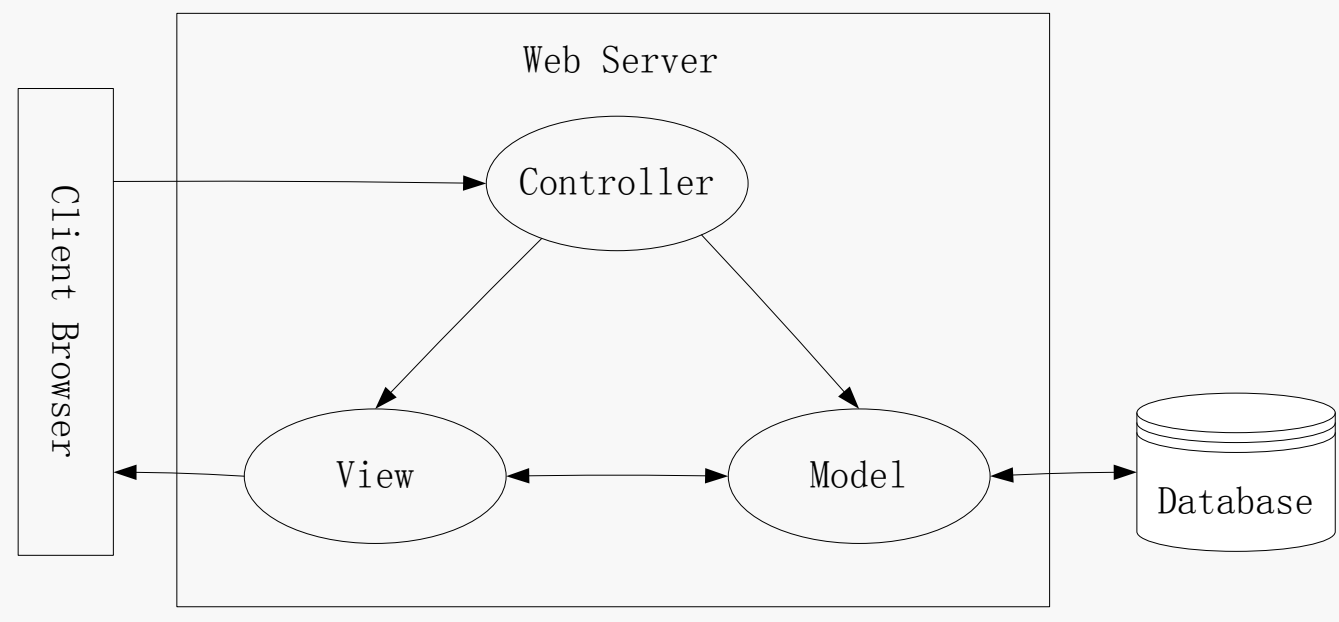

Figure 2 MVC architecture diagram

The MVC architecture mentioned above can basically meet the needs of the business logic development process. In addition, the framework provides a mechanism called the component for code combination and encapsulation which will be used multiple times in the system development process. Component is a container used to encapsulate common function, it has many convenient features preset in the framework, and using the component allows developers to quickly develop system function.

In addition, as the $\mathrm{B} / \mathrm{S}$ structure information system, display information on the browser, a lot of HTML/JAVASCRIPT codes are usually needed. According to the characteristics of information system, the framework system encapsulates many commonly used components such as form, sheet and so on for the front end developers.

The database access. In the framework, the system data is mainly managed through database. Therefore, for the database operation in the development process will make up a large part of the whole system. The database operation generally fall into two categories. One kind is simple data read and write operations, while the other kind is complex data query operation. The framework provides operation interface for the two types of operations. Developers can choose according to the demand [5].

The SQL mode. The SQL mode allows users to directly write SQL statements to the database. The interface of the framework in this pattern provides functions such as parameters filter and the result packaging. The developers using this pattern can be unrestricted flexible using most of the database functions provided.

The object-oriented model. The object-oriented model (Object Oriented) map the tables in the database to the classes. Each row in a table is mapped to the corresponding object, and each field is mapped to the object's properties. These classes are the model classes of the MVC code organization structure. So, adding and deleting operations to a database table in object-oriented 
mode will correspond to the creating deleting and modifying objects. In addition, the relationship between database tables can also be said in the form of object properties.

\section{References}

[1] Information system, Http://en.wikipedia.org/wiki/Information_system, 2014-06-12.

[2] Somerville, Cheng Cheng. Software engineering [M]. Mechanical industry publishing house, 2007-01-01.

[3] Chen, Hui-liang Duan. Design and implementation of general web information system based on J2EE framework [J]. Computer system application. 2011 (1) : 6-9.

[4] Xiao-li Zhang. Design and implementation of the Web OA system based on MVC pattern [J]. The computer technology and development. 2012 (22) : 63-66.

[5] Zi-chao Li. Design and prototype implementation of general framework for information system based on B/S structure [D]. Beijing: Beijing university of posts and telecommunications, 2015. 